\title{
TOXIC AND PHOTODYNAMIC EFFECTS OF TOLUIDINE BLUE ON LIVING BULL SPERMATOZA ${ }^{1}$
}

\author{
C. VAN D U IJ N, J R. \\ Biophysics Department, "Schoonoord", Research Institute for Animal Husbandry, \\ Hoogt 10, Utrecht, The Netherlands \\ Received July 10, 1961
}

Ix continualion of a comparative study of the effects of vital stains and fluorochromes on bull spermatozoa [3-6] by means of Rikmenspoel's photoelectric method $[12,13,15,16]$, the metachromatic basic dye toluidine blue has been investigated.

Toluidine blue is being used extensively in histo- and cytomicrochemistry for demonstration of metachromatic substances [9,10], for which purpose it may also be used as a vital stain for subject material suitable for use with this technique. For cells in tissue culture vital staining was found to be superior to staining of fixed material, the latter not showing metachromasia [2], even after fixation with the basic lead acetate-formal fixative especially recommended for demonstration of this phenomenon [9]. Vital staining of cells was performed by adding a drop of 0.5 per cent aqueous toluidine blue solution to the culture medium, removing the medium after a few minutes and washing with warm $\left(37^{\circ} \mathrm{C}\right)$ tyrode. If the cells were allowed to remain in contact with excess toluidine blue, when after prolonged observation cell death would occur, cytoplasmic and nuclear nucleoproteins would also acquire metachromasia, whereas in the intact cell $\beta$-metachromasia was restricted to cytoplasmic particles, the nucleoproteins appearing light blue $[2]$. With respect to spermatozoa, results obtained with vital methods were also superior to those using fixed material [1]. Wislocki did not succeed in obtaining any metachromatic staining with fixed spermatozoa of deer [17] or with those of men [18], while Friedlaender and Fraser reported negative results with washed and fixed ram sperm [8]. Contrary to these observations, I found positive metachromatic staining of human spermatozoa under a variety of conditions [ 1$]$, the best results being obtained by adding a tiny drop of 0.5 per cent toluidine blue solution to a drop of semen. Purple metachromasia, with a shift to the red, was observed in the region of the postnuclear cap and the head cytoplasm, the outer layer staining less intensely

\footnotetext{
1 This is the 139th publication of "Schoonoord", Research Institute for Animal Husbandry.
} 
and the middle-piece and tail only faintly staining. The nucleus did not show any appreciable staining. Post-vital staining did not yield different results.

In living bull spermatozoa essentially similar results were obtained. $\beta$-Metachromasia could be observed in the regions of the post-nuclear and acrosomal caps, whereas no penetration of dye into the nucleus could be established. Measurements of the mean velocity of bull spermatozoa and the number of specimens moving normally showed a definite, adverse effect of toluidine blue, mainly due to photosensitization.

\section{MATERIALS AND METHODS}

Freshly ejaculated semen from Dutch-Friesian and red-and-white Meuse-RhineY'ssel bulls was used after a two-step dilution procedure with ultramicroscopically clear egg yolk-sodium citrate buffer [11]. All details of the procedures of semen handling and the measuring methods were the same as described previously with respect to the investigations on the effect of diazine green [6], the only exception being that for determining the effect of illumination a dye concentration of only 4 p.p.m. (1:250,000) was used. The dye used was labelled "Toluidinblau nach Hoyer", manufactured by E. Merck A. G., Darmstadt.

\section{RESULTS}

\section{Experiments with varying dye concentration at constant dosage of illumination}

The concentrations used ranged from 2-200 p.p.m. At each concentration generally two recordings were made: the first one directly following mixing of the semen with the dye-containing diluent and the second one about $24 \mathrm{hr}$ later, the diluted semen with dye addition having been stored in the dark in a Dewar vessel with ice. In order to determine any toxic effect of the dye per se, the rates of velocity decrease with time $-\partial \bar{v} / \partial t$ and the rates of decrease of the numbers of spermatozoa moving normally were calculated from the results. The rates of decrease of the mean velocity and of the number of normally moving sperms were plotted as functions of the toluidine blue concentration and statistically tested for slope. With respect to $-\partial \bar{v} / \partial t$ the result was not significant.

The number $N$ per unit volume of spermatozoa moving normally has been found to decrease exponentially with time during the limited periods actually investigated $[12,13,14]$, according to:

$$
N_{t}=N_{o} \cdot e^{-k t} \text {, }
$$


yielding a straight-line relationship on a semi-logarithmic plot. Whether this relationship would hold over an extended range is doubtful, since according to it the number of moving specimens would only become zero at infinite time, whereas in reality the last specimen becomes immotile at a very finite

Fig. 1.-Half-life periods of the numbers of bull spermatozoa swimming normally, as a function of toluidine blue concentration. Averages of three experiments, with standard errors.

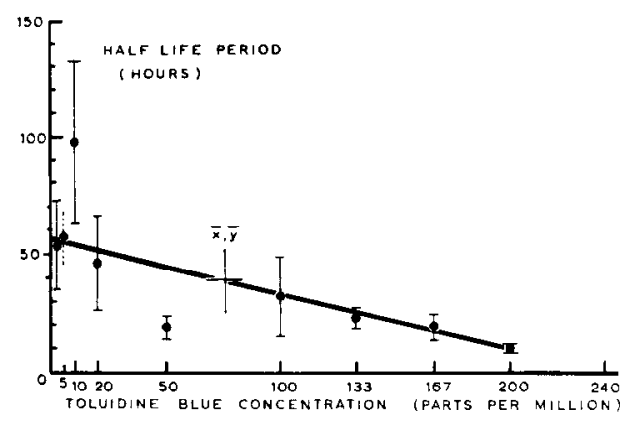

Fig. 2.-Averages of the numbers of bull spermatozoa swimming normally $(\bar{N})$ and their mean velocities $(\overline{\bar{v}})$ as a lunclion of toluidine blue concentration, expressed as percentages of the initial values at zero dye concentration. Four ejaculates from four different bulls, each measured twice. Upper part: Theoretical curves calculated after Freundlich type of eq. (2); lower part: Theoretical curves calculated after Langmuir type of eq. (3), showing better fit of this equation to the data than the equation derived from the Freundlich adsorption isotherm.

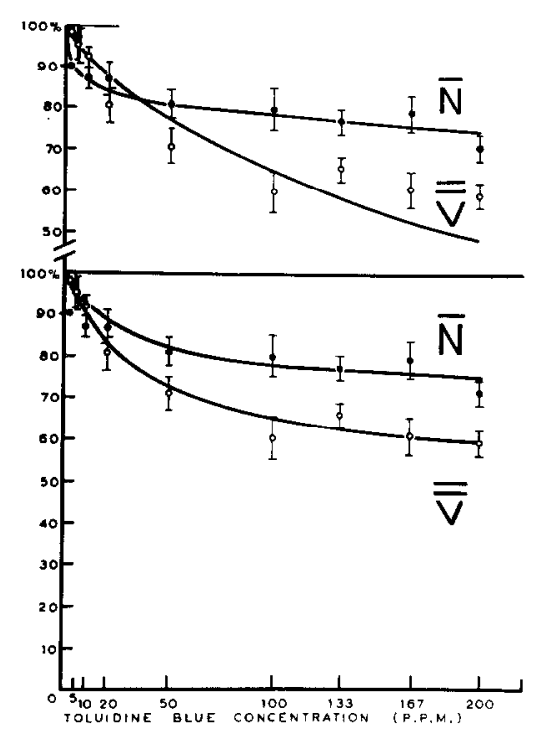

time indeed. However, eq. (1) is sound over a range of at least two or three half-life periods, allowing the use of half-life periods $t_{\frac{1}{2}}(N)$ as a measure for the rate of decrease. Fig. 1 shows the relationship between the half-life periods and dye concentration after storage in darkness. The slope of the regression line is significantly different from zero (1 per cent level), indicating that toluidine blue also exerts an adverse effect in darkness.

However, the slopes of the regression lines obtained after straight-line transformation of the data according to eq. (3), derived from Langmuir's 
adsorption isotherm by assuming strict proportionality between biological effect and adsorbed mass per unit area [6], did not differ significantly between measurements taken directly and after $24 \mathrm{hr}$ storage. From these results it may be concluded that determination of half-life periods is more sensitive for detecting intrinsic toxicity than testing slope differences of the dosage-effect curves for $\bar{v}$ and $N$.

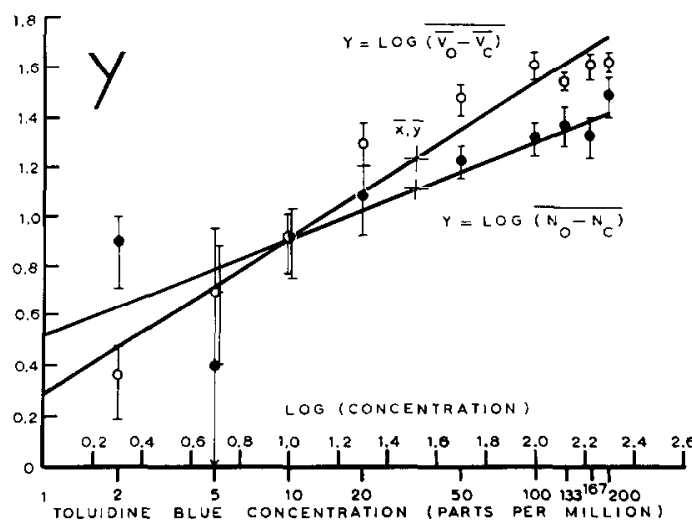

Fig. 3.--Straight-line transformation according to eq. (2) of the data represented in Fig. 2 (equation derived from Freundlich adsorption isotherm).

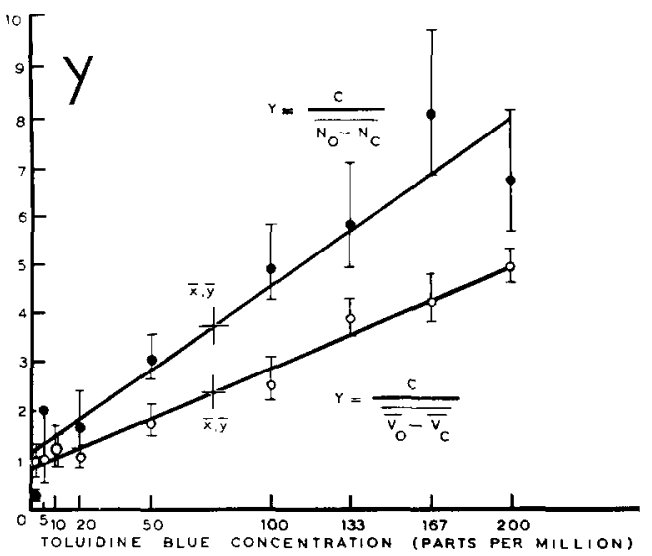

Fig. 4.- Straight-line transformation according to eq. (3) of the data represented in Fig. 2 (equation derived from Langmuir adsorption isotherm).

Fig. 2 gives the combined data obtained for the number of spermatozoa moving normally and their mean velocity, expressed as percentages of the initial values at zero dye concentration, as a function of toluidine blue concentration. The upper graph shows the theoretical curves calculated from the data on the basis of eq. (2), founded on the empirical Freundlich adsorption isotherm, whereas the lower graph gives the curves corresponding to the theoretically founded eq. (3) derived from Langmuir's adsorption isotherm $[6]$. 


$$
\begin{aligned}
& \bar{v}_{\mathrm{c}}=\bar{v}_{o}-k \cdot C^{(p / q)} \\
& \bar{v}_{c}=\bar{v}_{o}-\frac{k \cdot C}{C+a}
\end{aligned}
$$

$N_{\mathrm{c}}=N_{o}-k \cdot C^{(p / q)}$

$$
N_{c}=N_{o}-\frac{k \cdot C}{C+a}
$$

where $\bar{v}_{c}=$ mean velocity at concentration $C, \bar{v}_{o}=$ mean velocity without dye addition, $N_{c}$-number of spermatozoa moving normally, at concentration $C$, preferably expressed as percentage relative to the number at zero concentration, $N_{o} ; k, p, q$, and $a$ are constants; $p / q$ represents the product of the Freundlich exponent $1 / q$ with a proportionality constant $p$, which may or may not be dependent on other conditions, such as the illumination level; $k$ in (3) represents the specific adsorptive power of the adsorbing surface with respect to the adsorbed substance.

From these curves, as well as from the straight-line transformations (Figs. 3-4), it can be seen that the Langmuir type of eq. (3) may be regarded as a better fit to the data than the empirical eq. (2). Further, it is clearly demonstrated that numerical values of constants in the same type of equation are different with respect to $\bar{v}$ and to $N$, as indicated by different slopes and different heights of intersect with the axis, consequently the effect on longevity of the spermatozoa is different from that on swimming velocity of the surviving specimens.

\section{Effect of toluidine blue on the velocity frequency distribution}

The standard deviation (biological spread, not error) of the velocity frequency distribution of the controls was $29 \mu / \mathrm{sec}$, in good agreement with the values of 30 found in previous investigations $[6,7]$. The mean standard deviation of the samples to which toluidine blue had been added in concentrations from $2-200$ p.p.m. was $25.2 \pm 0.9$ (s.E.) $\mu /$ sec. This difference is highly significant $(0.001<P<0.01)$.

Fig. 5.- Normalized velocity frequency distributions of normal bull spermatozoa with toluidine blue $100-200$ p.p.m. compared with control without dye addition. The distributions in the range 100-200 p.p.m. of dye do not differ significantly among themselves. Pooled data of four ejaculates from four different bulls.

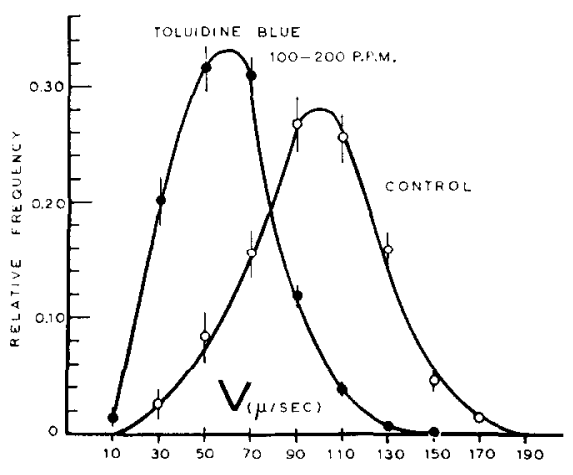


After $24 \mathrm{hr}$ storage the results were $26.6 \mu /$ sec for the controls and $23.9 \pm$ $0.86 \mu /$ sec for the samples with dye addition. This difference was also significant, but less pronounced $(0.01<P<0.02)$. The mean standard deviation of the samples with dye addition measured after $24 \mathrm{hr}$ storage was not significantly different from the value obtained from the first measurements: $P \sim 0.30, \Delta \xi \bar{\sigma}=1.32 \pm 1.26 \mu /$ sec.

The type of change of the velocity frequency distribution curves (Fig. 5) is similar to that produced by diazine green (Janus green) [6].

\section{Experiments with varying light dosage at constant dye concentration.}

The photodynamic activity of toluidine blue was found to be very high. At concentrations $\geqslant 10$ p.p.m. the numbers of moving specimens decreased so fast under influence of illumination that it was impossible to take a sufficient number of recordings (for technical reasons it is difficult to take recordings of one sample at intervals less than about $15 \mathrm{~min}$, or at best $10 \mathrm{~min}$ ). As in previous investigations [4-6], the light source employed was a $150 \mathrm{~W}$, $220 \mathrm{~V}$ incandescent frosted lamp with an internal mirror, and the temperature of the samples during irradiation was $0^{\circ} \mathrm{C}$. Under these conditions a suitable

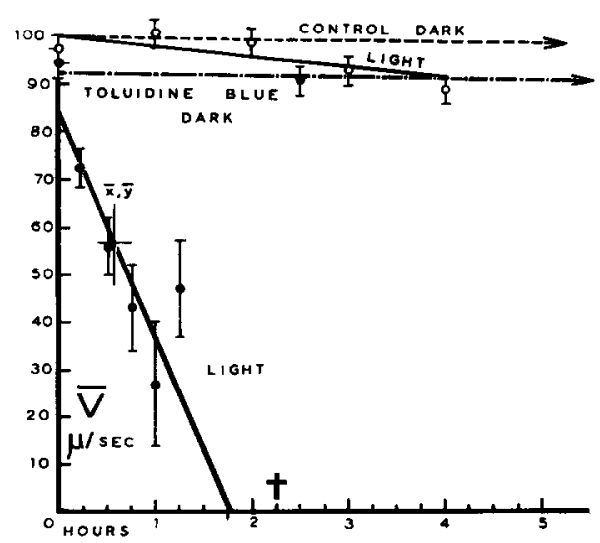

Fig. 6 .

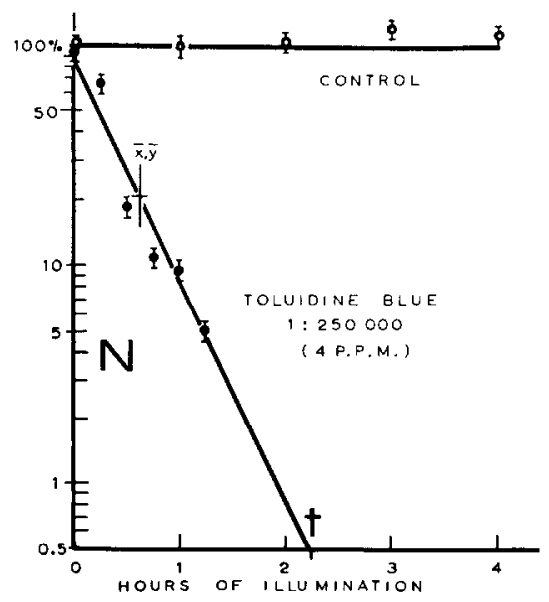

Fig. 7.

Fig. 6.--Mean velocity of bull spermatozoa as a function of time, with and without toluidine blue, kept in darkness and under illumination with white light (at $0^{\circ} \mathrm{C}$ ), respectively. At $\dagger$ no actively swimming specimens could be observed under the microscope.

Fig. 7.-Semi-logarithmic plot of the number per unit volume, of bull spermatozoa moving normally, under illumination with white light (at $0^{\circ} \mathrm{C}$ ), with and without toluidine Ilue. Initial value of the control taken as 100 per cent. At $\uparrow$ no moving specimens could be observed under the microscope. 
concentration was 4 p.p.m. $\left(1: 250,000\right.$ or $\left.1.3 \times 10^{-8} M\right)$. With the samples kept in darkness there was no demonstrable difference between the semen with toluidine blue and the control with respect to the rate of decrease of velocity and the rate of decrease of the number of spermatozoa moving normally, except some shift to a lower level, which can be accounted for by the effect of optical sensitization by the dye during the period of recording in the dark field microscope with an $8 \mathrm{~V}, 6 \mathrm{~A}$ incandescent lamp as the light source.

In the illuminated samples the number of spermatozoa moving normally and their mean velocity decreased extremely more rapidly in presence of toluidine blue than in the controls without dye addition (Figs. 6-7).

\section{DISCUSSION}

The effect of decreasing half-life periods of the number of normally noving spernatozoa with increasing dye concentration on storage in darkness indicates an intrinsic toxicity of toluidine blue. The theoretical possibility that this effect might still be due to photosensitization is excluded by the procedure followed, where the semen is stored in tubes of brown glass throughout, the dilution medium containing toluidine blue being pipetted on top of the samples in these tubes. Only during the short moment of transport of a drop of the sample with dye to the microchamber is it subjected to the general laboratory illumination level, which however is so much lower than the dosage received during the measuring procedure, that it cannot account for the difference.

It may be expected that a significant difference in slope of the $\bar{v}=f(C)$ and $N=f(C)$ functions (after straight-line transformation) would appear if a larger number of data were available; with the material in hand the mean slope difference for the velocity data as function of the concentration measured directly and after $24 \mathrm{hr}$ storage was just at the border of significance ( $P \sim$ 0.05). However, it was not considered worth while to investigate this further at the moment since the result obtained with respect to the half-life period of the number of specimens moving normally is in itself sufficient evidence for a toxic effect in darkness, whereas the scope of the investigations as a whole centres on photodynamic effects, which are the more important in the usual short-term observations.

The adverse effect of photosensitization by toluidine blue as demonstrated by the present investigations greatly exceeds those observed with acridineorange $[3,4]$, coriphosphine, rhodamine $B$, primuline, methyl green $[\bar{\jmath}]$, 
diazine green (Janus green) [6] and fluorescein sodium (uranin) (Van Duijn, unpublished), under the same conditions.

Unlike diazine green, the effect of toluidine blue is not identical with respect to decrease of velocity and the decrease of the number of specimens moving normally; the mean velocity decreases much faster than the number of spermatozoa maintaining normal movement. This indicates a less simple process than with diazine green, where it appeared that the total adverse effect can be attributed to one single site of attack. With toluidine blue we have to explain why velocity is affected more than maintenance of normal movement. Although admittedly evidence is lacking, it might be that adsorption to some internal part of the cell, especially of the locomotive centres in the midpiece, is the major effect responsible for both decrease of number and of the mean velocity of the survivors that still show normal movement. This major effect might be a one-site attack, like that of diazine green. To explain the faster decrease of velocity with increasing dye concentration, a second effect would have to be assumed affecting velocity only. Such an effect could well be a change in the outer layers of the head (acrosome, post-nuclear cap, or membrane), where the main part of observable dye adsorption takes place, leading to increased fluid friction. To test this hypothesis special investigations of a different kind would be required, such as measurements of the sedimentation velocity of stained and unstained immobilized specimens.

As a vital stain toluidine blue is only suitable for qualitative observation with regard to morphology and cytochemistry. For evaluation of motility it is entirely unsuitable owing to the extreme photodynamic effect.

\section{SUMMARY}

1. The effects of vital staining with toluidine blue on the total number of bull spermatozoa moving normally and on their mean velocity and velocity frequency distributions have been investigated with Rikmenspoel's photoelectric method.

2. A significant decrease of the mean half-life period of the number of spermatozoa moving normally with increasing concentration, after storage in darkness near $0^{\circ} \mathrm{C}$, indicates an intrinsic toxicity of toluidine blue, apart from its activity as an optical sensitizer.

3. Under illumination, toluidine blue acts as a most powerful photosensitizer, even in concentrations of only $1-4$ p.p.m. $\left(0.3-1.3 \times 10^{-8} M\right)$. The effect is greater with respect to the mean velocity of the spermatozoa with 
normal movement than to the total number maintaining normal movement suggesting at least two separate ways of action.

4. The relationship between effect and dye concentration (at constant illumination level) can be most accurately described by the equations:

$$
\bar{v}_{c}=\bar{v}_{o}-\frac{k \cdot C}{C+a} \text { and } N_{c}=N_{o}-\frac{k \cdot C}{C+a},
$$

where $\bar{v}_{c}$ and $N_{c}$ are the mean velocity and the number of specimens per unit volume, moving normally and at concentration $C$, respectively; $\tilde{v}_{o}$ and $N_{o}$, the corresponding values without dye addition; $k, a$ are constants. These equations are derived from the Langmuir adsorption isotherm by assuming strict proportionality between biological effect and adsorbed mass of dye per unit of susceptible area. Equations derived from the empirical Freundlich adsorption isotherm fit the data less well.

5. The velocity frequency distributions become more skew with increasing dye concentration, wilh a significant decrease of the standard deviation and consequent rise of the top value of the normalized curve.

The author is indebted to Miss Elisabeth Muilwijk for her skillful and excellent assistance during the course of these investigations.

\section{REFERENCES}

1. Duinn, C. vai, Jr., J. Roy. Microscop. Soc. 74, 69 (1954).

2. - Mikroskopie 10, 75 (1955).

3. — Nature 187, 1006 (1960).

4. -_ Nature 189, 76 (1961).

5. — - Proceedings IVth Int. Congress Animal Reprod. The Hague, June 5-9, 1961 (in press).

6. - Exptl. Cell Research 25, 120 (1961).

7. Duijn, C. van, Jr. and Rikmenspoel, R., J. Agric. Sci. 54, 300 (1960).

8. Friedlaender, Marlene H. G. and Fraser, M. J., Exptl. Cell Research 3, 462 (1952).

9. Glick, D., Techniques of Histo- and Cytochemistry. Interscience Publishers, New York and London, 1949.

10. Pearse, A. G. Everson, Histochemistry. Churchill Ltd., London, 1954.

11. Rrkmenspoel, R., Experientia 13, 124 (1957).

12. - Photoelectric and cinematographic measurements of the "motility" of bull sperm cells. Thesis, Utrecht, 1957.

13. — J. Agric. Sci. 57, 399 (1960).

14. Rikmenspoel, R. and Duijn, C. van, Jr., Tijdschr. Diergeneesk. 85, 1002 (1960).

15. Rikmenspoel, R. and Herpen, G. vas, Phys. Med, Biol. 2, 54 (1957).

16. Rrkmenspoel, R., Herpen, G. van, Dam, G. C. van, and EiJkhout, P., Tijdschr. Diergeneesk. 85, 909 (1960).

17. Wislocki, G. B., Endocrinology 44, 167 (1949).

18. - Anat. Record 108, 645 (1950). 